\title{
Féeries
}

Études sur le conte merveilleux, XVII ${ }^{\mathrm{e}} \mathrm{XIX} \mathrm{X}^{\mathrm{e}}$ siècle

5 | 2008

Le rire des conteurs

\section{Le rictus moral de Marmontel}

\section{Nicolas Veysman}

\section{OpenEdition}

\section{Journals}

Édition électronique

URL : http://journals.openedition.org/feeries/685

DOI : $10.4000 /$ feeries. 685

ISSN : 1957-7753

Éditeur

UGA Éditions/Université Grenoble Alpes

Édition imprimée

Date de publication : 1 juin 2008

Pagination : 115-132

ISBN : 978-2-84310-123-6

ISSN : $1766-2842$

\section{Référence électronique}

Nicolas Veysman, « Le rictus moral de Marmontel », Féeries [En ligne], 5 | 2008, mis en ligne le 01 septembre 2009, consulté le 08 septembre 2020. URL : http://journals.openedition.org/feeries/685 ; DOI : https://doi.org/10.4000/feeries.685

Ce document a été généré automatiquement le 8 septembre 2020.

(c) Féeries 


\title{
Le rictus moral de Marmontel
}

\author{
Nicolas Veysman
}

$1 Q_{U^{\prime} E S T-C E} Q U^{\prime} U N$ CONTE? Celui qui cherche une définition du conte au XVIII ${ }^{\mathrm{e}}$ siècle ou un discours sur sa place dans la hiérarchie des genres littéraires ouvrira sans grand succès les différents arts poétiques de l'âge classique et autres cours de belles lettres, car tous refusent une dignité littéraire à ce petit genre méprisé des théoriciens, a fortiori lorsqu'il est écrit en prose. Cette carence théorique et poétique du conte au XviII ${ }^{\mathrm{e}}$ siècle est d'autant plus paradoxale que le siècle des Lumières, s'il fut celui de la philosophie, passe aussi pour être celui du conte ${ }^{1}$. C'est en effet à ce moment qu'apparaissent plusieurs formes: après le conte de fées sont inventés le conte oriental, le conte parodique, le conte libertin, le conte moral, le conte philosophique, le conte historique, le conte fantastique et le conte immoral; mais rares sont les conteurs qui ressentent la nécessité de définir le genre qu'ils pratiquent. Il est vrai que les créateurs de ces genres nouveaux, qui, comme Perrault, Galland, Hamilton, Crébillon fils, Marmontel ou Voltaire, en offrent bien souvent les plus parfaites illustrations, ont aussi le mérite d'accompagner leurs créations de discours théoriques dans lesquels ils s'expliquent sur ce qu'est pour eux leurs contes. Mais en sait-on davantage sur ce qu'est le conte au xvIII siècle?

\section{Le conte moral : un « conte à rire »?}

2 Pour une saisie d'ensemble du genre, nous n'avons d'autres choix que d'ouvrir les dictionnaires de langue. Le très officiel Dictionnaire de l'Académie, par exemple, formule en 1694 une définition qui restera inchangée dans les éditions ultérieures: «Narration, récit de quelque adventure, soit vraye, soit fabuleuse \& plaisantes ${ }^{2} »$. Sont ensuite mentionnés, à titres d'exemples, plusieurs emplois du terme: «beau conte», «conte pour rire», «conte à dormir debout», «conte en l'air», «conte gras» et «sot conte». Nous sommes évidemment tentés de croiser cette typologie lexicale avec la liste des sousgenres du conte que nous avons dressée plus haut, et, si nous avions l'ambition de mener à bien ce travail, nous aurions certainement à nous demander quelles formes mériteraient d'être appelées «contes à rire». Nous nous en tiendrons ici au seul 
exemple du conte moral, et plus encore aux seuls contes moraux de Marmontel, pour nous demander s'ils sont ou ne sont pas des «contes à rire».

Remarquons d'abord que Marmontel confirme nos allégations préliminaires sur les conteurs, puisqu'il fut tout à la fois le créateur, le meilleur auteur et le théoricien du conte moral. Nous trouvons dans l'article «Conte» de ses Éléments de littérature le détail de ses idées sur le genre. Parmi toutes les notions que convoque le conte, celle de gaieté est selon lui primordiale et elle lui apparaît comme un principe invariant du genre: «En général, ce qu'il y a de plus précieux et de plus rare dans l'art de conter, ce n'est pas la parure des grâces, mais leur négligence, ce n'est pas le mordant de la plaisanterie, mais la finesse et surtout la gaieté ${ }^{3} »$.

4 En effet, qu'il soit en vers ou en prose, merveilleux ou vraisemblable, un conte est pour lui un récit enjoué destiné à étendre sa bonne humeur dans l'esprit des lecteurs: «Le conte est à la comédie ce que l'épopée est à la tragédie, mais en petit, et voici pourquoi. L'action comique n'ayant ni la même importance ni la même chaleur d'intérêt que la tragédie, elle ne saurait nous attacher aussi longtemps lorsqu'elle est en simple récit.» Cette nature comique du conte est si importante aux yeux de Marmontel qu'il accorde au comique une parfaite prééminence sur la moralité du propos. La chose est d'autant plus aisée que le conteur n'oppose jamais le rire à la morale. Au contraire, il les articule en associant dans le conte les notions de «situation» et de "caractère» qui relèvent aussi bien du comique que du pathétique. Remarquez dans ce passage l'habile indétermination que cultive Marmontel à propos du registre visé:

La partie la plus piquante du conte sont les scènes dialoguées. C'est là que les mœurs peuvent être vivement saisies, finement indiquées, délicatement nuancées, et qu'avec des touches légères, mais brillantes de vérité, un peintre habile peut produire des groupes animés et des tableaux vivants. Mais selon que ces groupes seront mieux composés, ils donneront eux-mêmes du dialogue un mouvement plus vif, une vérité plus exquise. C'est toujours par les situations que les caractères sont mis en jeu; et c'est le jeu des caractères et à leur singularité que tient l'intérêt de la scène ${ }^{4}$.

Il n'est jamais question du conte moral dans cet article, mais Marmontel en écrit une définition à l'encre sympathique qui n'apparait qu'à l'envers de la définition du conte comique; car tout nous porte à croire que le conte moral est une parfaite inversion du «conte à rire».

6 Pour lors, l'inventeur du conte moral ne voit aucune antinomie entre la visée comique et la visée morale du conte, mais la chose ne va pas de soi, et le rire va bientôt poser problème au moraliste. Un problème qui est rendu manifeste par le recul toujours plus grand, et jusqu'à l'extinction, du comique dans les contes de Marmontel, sans que l'on comprenne d'emblée pourquoi. On peut certes invoquer, comme nous le ferons d'abord, l'évolution du comique $d u$ XviII ${ }^{e}$ siècle sur laquelle le conteur règlerait sa production morale. Le rôle joué par l'émergence d'un nouveau comique est incontestable, mais il nous faudra aussi considérer l'existence de causes internes à l'éviction morale du rire dans l'œuvre marmontélienne. Autant de questions qui nous conduisent à douter que les contes moraux de Marmontel soient véritablement des contes et à affirmer que seuls les contes immoraux sont des contes, des «contes à rire».

7 On rit peu dans les contes moraux de Marmontel. Plus exactement, de 1755 à 1793, des anciens aux nouveaux contes moraux, les personnages comme les lecteurs y trouvent toujours moins matière à rire. Il existe pourtant une véritable veine comique des premiers contes, mais elle s'épuise à partir de 1758, lorsque Marmontel fait paraître son 
premier «conte moral» intitulé Les Deux Infortunées. Peut-être serait-il bon de rappeler que les premiers contes publiés par Marmontel dans le Mercure de France ne furent pas des «contes moraux», mais des «histoires» ou des «anecdotes», qui ne reçurent que plus tard, en 1761, leur appellation générique définitive. Tout se passe donc comme si la faveur grandissante du syntagme «conte moral» chassait progressivement et inexorablement la gaieté hors du conte marmontélien. Étrange paradoxe: seraient donc de véritables contes (des récits enjoués) ceux qui ne reçurent pas primitivement cette dénomination. Une seule exception notable: Le Scrupule, baptisé seulement «conte» en 1757.

8 Autre paradoxe: en 1765, au moment d'offrir au public enthousiaste la collection complète de ses contes moraux, Marmontel rédige une préface où il accorde la plus grande place à... l'écriture comique. À en croire leur créateur, les contes moraux seraient la continuation, sur un mode narratif, de l'entreprise moliéresque de dénonciation des ridicules:

Engagé, il y a quelques années, à écrire sur la comédie, je cherchais dans la nature les règles et les moyens de l'art. Cette étude me conduisit à examiner s'il est vrai, comme on l'a dit, que tous les grands traits du ridicule eussent été saisis par Molière et par les poètes qui l'ont suivi. En parcourant le tableau de la société, je crus apercevoir que les combinaisons inépuisables des folies et des travers de tous les états, un homme de génie trouverait encore de quoi s'occuper. J'avais même recueilli quelques observations que je voulais proposer aux jeunes poètes, lorsque M. de Boissy, mon ami, me demanda quelques morceaux de prose à insérer dans le Mercure. Il me vint dans l'idée de mettre en œuvre, dans un conte, l'un des traits de ma collection $[. . .]^{5}$.

Cédant à l'autorité de la préface auctoriale, il nous faut convenir que cette inspiration moliéresque est présente dans tous les contes. Comme chez Molière, d'autres registres affleurent derrière le rire que provoque la satire, notamment les registres pathétique et tragique. D'abord minoritaires, ces derniers vont connaitre une inflation grandissante dans les contes moraux jusqu'à devenir la composante exclusive des nouveaux contes moraux écrits dans les années 1790. Ainsi, même si l'opposition entre description des mœurs et prescription moralisatrice a pu servir de principe explicatif pour l'évolution du genre, nous lui préférons ici l'opposition entre conte comique ou «conte à rire» et conte pathétique ou «conte à pleurer».

\section{Un nouveau rire?}

L'heure est venue pour nous de comprendre les causes de cette éclipse du comique dans les contes de Marmontel. Il est possible de l'expliquer par l'évolution parallèle de la scène dramatique, c'est-à-dire par l'invention du drame bourgeois. Il est vrai que les points de convergence sont nombreux entre les contes et le drame, mais n'oublions pas que ce n'est pas au drame mais à la comédie la plus classique que Marmontel fait référence dans sa préface de 1765 . Il est aussi possible d'invoquer, comme l'a fait très pertinemment Kate Astbury, l'influence de la littérature anglaise sur l'écriture marmontélienne du conte moral, mais peut-on imputer à l'anglomanie naissante la responsabilité d'un reflux du comique chez notre conteur? La faveur nouvelle des belles anglaises éplorées ne doit pas nous faire oublier quelles sont les tensions qui sont au cœur du conte moral comique. 
11 En effet, sans mésestimer les charmes puissants du pathos sur les lecteurs de la moitié $\mathrm{du} \mathrm{XVIII}^{\mathrm{e}}$ siècle, nous pouvons supposer que l'inflexion pathétique du conte moral est aussi déterminée par une restriction morale des ressources comiques, qui conduit à une éviction pure et simple du rire. La cause: l'immoralité foncière du rire satirique fondé, comme l'écrivait jadis Molière, sur «quelque discordance», sur une «apparence différente du fond» chez ceux, perfides séducteurs, parents autoritaires et pédants insupportables, qui sont sommés de rejoindre ce «juste milieu dans lequel se trouve la justice, la raison et la vérité». On rit alors, mais de qui rit-on au juste? Sur cette question, Marmontel fait une judicieuse analyse du phénomène, à lire dans l'article «Comique» de l'Encyclopédie, repris dans ses Éléments de littérature:

L'effet du comique résulte de la comparaison qu'on fait, même sans s'en apercevoir, de ses mœurs avec les mœurs qu'on voit tourner en ridicule, et suppose entre le spectateur et le personnage visible une différence avantageuse pour le premier. Il arrive pourtant quelquefois que l'on rie de sa propre image, même en s'y reconnaissant; cela vient, ou du plaisir secret qu'on a de se croire plus adroit qu'un autre à échapper au ridicule, ou d'une duplicité de caractère qui s'observe encore plus sensiblement dans le combat des passions, où l'homme est sans cesse en opposition avec lui-même. On se juge, on se condamne, on se plaisante comme un tiers; et l'amour-propre y trouve son compte ${ }^{6}$.

12 Marmontel est peu favorable à la vis comica et c'est sur des arguments moraux qu'il assoit son réquisitoire. Comprenons bien ce que dit Marmontel: il reproche certes au rieur d'être imbu de cette "différence avantageuse» qui flatte son "amour-propre», mais le comique est moins à ses yeux le fait d'un caractère que le propre d'une «relation» immorale. Le rieur est donc moins celui qui a l'humeur moqueuse que celui qui est placé en situation de rire de son semblable. La différence est importante, parce qu'elle fait du rire non plus l'expression d'une humeur particulière, mais un enjeu moral qui engage l'humanité tout entière. Le rire de chaque homme nous dit quelque chose de la relation qu'il entretient avec les autres hommes; le rire nous dit quelque chose de la moralité de chacun d'entre nous: "Chaque homme méprisera dans son semblable ceux dont il se croira exempt et prendra un plaisir malin à les voir humilier; ce qui assure à jamais le succès du comique qui attaque les mœurs générales.» Si le rieur est immoral, c'est à cause de la relation dissymétrique établie par le dispositif comique, où le plaisir est toujours éprouvé aux dépens d'une victime ridicule: nous rions des faiblesses qui ne sont pas les nôtres, alors que l'homme sensible s'émeut de souffrances qui sont ou pourraient être les siennes, comme c'est le cas dans le drame bourgeois qui, écrit Marmontel dans son article «Drame», «a renoncé au ridicule» et auquel il ne «reste plus que les moyens de la tragédie, la terreur et la compassion».

13 Cette tension entre une immoralité intrinsèque de la relation comique et une moralité confondue avec la seule compassion vertueuse est présente dès les premiers contes et va, à partir de 1758, se résoudre en un divorce entre deux formes simples: d'une part le conte moral, exclusivement pathétique et larmoyant, d'autre part le conte immoral, qui accueille le «rire décapant, cruel, mécanique, extérieur et supérieur, plein de mépris et de répulsion», selon les termes de Jean Goldzink.

Marmontel se serait-il mépris sur la nature du comique moliéresque lorsqu'il choisit en 1765 l'auteur du Tartuffe comme modèle pour ses contes? pourquoi ériger Molière comme autorité, alors même qu'il a pris ses distances avec la verve satirique du maître et qu'il s'est engagé dans le sillon de la comédie larmoyante et du drame bourgeois? 
15 Molière a encore sa place dans la préface de 1765, parce que Marmontel entreprend moins d'abandonner la veine comique traditionnelle que de la convertir aux exigences nouvelles de la morale des Lumières. À ce titre, le conteur moraliste est toujours celui qui relève les ridicules de ses contemporains, mais il s'agit dorénavant d'exciter la compassion plutôt que les rires.

16 Marmontel va ainsi mener de front les deux esthétiques, celle immorale de la satire des ridicules et celle morale de la narration sensible des épreuves de la vertu. Certains titres, comme le couple formé par La Bonne Mère et La Mauvaise Mère, pourraient nous faire croire que le conteur verse tantôt dans l'une, tantôt dans l'autre esthétique, mais il n'en est rien. Il peut certes arriver que la charge satirique se fasse plus violente et qu'elle menace la moralité du propos; on sait que les années 1760 sont riches en attaques personnelles, que Palissot et l'abbé Morellet croisent la plume, et Marmontel semble un temps séduit par le portrait charge dont il offre un savoureux exemple dans Le Philosophe soi-disant où il contrefait Rousseau sous les traits d'un goujat pédant et vénal; mais convenons-en, les exemples sont rares et bien plus souvent Marmontel contient sa verve satirique.

17 La gageure est ailleurs. Elle réside dans un improbable équilibre que Marmontel s'efforce d'établir dans chaque conte entre le rire et les larmes, de parvenir à une parfaite coexistence des deux veines comique et pathétique. Marmontel y parvient en intégrant la vis comica dans le dispositif pathétique, c'est-à-dire en faisant de la dénonciation des ridicules une modalité de l'éloge sentimental des vertus. On reconnaît ordinairement peu de mérite à Marmontel conteur, mais nous pouvons lui reconnaître celui de s'être frotté à l'art difficile d'un Térence, qui consiste à mêler la cruauté des ris à la douceur des larmes. Art difficile, car menacé par la contradiction et l'inefficience, comme l'écrit Anne Coudreuse: «Le véritable pathétique, celui qui possède une réelle efficacité sur le destinataire, n'admet pas la distance, si minime fût-elle, parce que l'ironie, l'humour ou même un très léger décalage de ton peuvent s'immiscer dans cet écart, portant un coup fatal au pouvoir d'émotion, sinon de commotion, du pathétique sur le lecteur, puisque cette potentialité de contagion émotive est liée à l'adhésion, voire à l'adhérence du discours pathétique à lui-même ${ }^{7}$ ». Marmontel a parfaitement conscience de cette tension qui rend si difficile la conciliation des deux registres et qui est au cœur de la mutation du comique moral des Lumières. Marmontel l'a même glosée dans un conte, Le Misanthrope corrigé, dont le seul titre souligne l'inspiration moliéresque et la «correction» morale du modèle. L'auteur des Contes moraux en résume l'intrigue dans sa préface: «En écrivant la comédie du Misanthrope, j'avançai il y a quelque temps que Molière, dans le personnage de Philinte, avait prétendu opposer à Alceste un homme du monde, et non pas un sage. Il m'est venu depuis dans la pensée d'essayer comment le Misanthrope aurait soutenu le contraste d'un homme vraiment vertueux. C'est ce faible essai que je donne sous le titre du Misanthrope corrigé». Le nouvel Alceste a les mêmes défauts que l'ancien, mais le voisinage du sage et vertueux M. de Laval amène le lecteur à éprouver de la compassion pour l'atrabilaire amoureux.

\section{Un rire dangereux}

Réhabiliter Alceste contre Rousseau, pleurer les malheurs du premier et rire des audaces du second, n'est-ce pas aller contre le sens de l'Histoire, à rebours des revendications des Lumières? Marmontel épouse le sentimentalisme de son siècle, mais 
quelle est sa position face à l'émergence de la conception militante de la philosophie au XVIII ${ }^{\mathrm{e}}$ siècle? L'étude du rire dans les contes moraux nous aide à apporter une réponse à cette question; nous disons même que le rire est la pierre de touche grâce à laquelle nous pouvons dire ce qu'est véritablement le conte moral.

Ce n'est pas un «conte à rire» mais un «conte à pleurer», le récit attendrissant de la vertu éprouvée; ce n'est donc pas un de ces contes voltairiens forts de leurs sarcasmes ironiques employés comme une arme puissante sur la scène publique et politique où s'invite le philosophe au midi du siècle, car l'univers du conte moral est aux antipodes de la guerre déclarée par les écrivains contestataires qui revendiquent un droit de penser sur les matières coutumièrement réservées à l'État: le droit, la religion, l'économie et la politique. En 1764 pourtant, Voltaire invitait Marmontel à plus d'audace et à plus de fantaisie, à écrire des «contes à rire»: «Vous devriez bien nous faire des contes philosophiques, où vous rendriez ridicules certains sots et certaines sottises, certaines méchancetés et certains méchants: le tout avec discrétion, en prenant bien votre temps, et en rognant les ongles de la bête quand vous la trouverez un peu endormie.» Mais ce combat n'est pas du goût de notre moraliste. Marmontel, ami plus compagnon de lutte des philosophes, saura, en prudent homme de lettres, se tenir à l'écart du scandale, au moins jusqu'en 1767, date à laquelle éclate l'«affaire Bélisaire». C'est à cette date et seulement à cette date que le moraliste rejoint, bien malgré lui, les rangs de la philosophie militante. À Bélisaire succéderont immédiatement Les Incas, écrits en 1767 mais publiés en 1777.

Marmontel n'aime pas le scandale. En somme, il cesse d'écrire des contes lorsqu'il est amené à jouer un rôle dans la «sphère publique bourgeoise» (Jürgen Habermas), c'est-àdire dans cet espace intermédiaire entre la sphère privée et la sphère étatique.

Le rire et son éviction du conte sont liés à cette économie de la fiction marmontélienne en prose entre 1755 et 1767. La restriction du comique, sa subordination au pathétique puis son extinction sont déterminées, disons-nous, par le mode de présence de la nouvelle sphère publique bourgeoise dans la fiction morale. Rien de plus normal lorsque l'on sait que l'opinion publique constitua une instance de censure morale avant de devenir, vers 1770 selon Keith Michael Baker, un nouveau fondement de l'autorité politique ${ }^{8}$.

Nous sommes d'abord tentés d'opposer la sphère privée des contes moraux écrits entre 1755 et 1765 à la sphère publique des romans composés en 1767, de sorte que nous aurions, avec la désaffectation du conte et le choix du roman, une traduction littéraire d'un engagement philosophique de Marmontel dans la sphère publique bourgeoise. Beau mouvement, mais les choses sont plus complexes, car, à y regarder de près, les sphères coexistent dès 1755 et jusqu'en 1767. Ce qui change, c'est la focalisation de l'écrivain sur l'une ou l'autre sphère: le conteur choisit pour scène principale la sphère privée, celle du couple et de la famille, tandis que le romancier s'attache au contraire à décrire une sphère publique à l'ombre de laquelle est furtivement évoquée la vie privée des personnages. En dépit de cette divergence, contes et romans ont en commun la même caractérisation des lieux, puisque, ici et là, la sphère privée est invariablement dévolue à toutes les formes du bien, et la sphère publique abandonnée aux puissances du mal. Il serait plus juste de dire que cette géographie morale est établie dès les contes et qu'elle est reconduite dans les romans avec la volonté d'explorer en 1767 les marges maléfiques de l'espace vertueux du conte. Marmontel écrit donc des romans lorsqu'il se résout à aborder la question du mal. Est- 
ce à dire que le mal est absent du conte? Il n'en est pas absent: il rôde, il menace et s'abat sur quiconque aura eu l'imprudence de quitter le cercle béni de la famille. Le conte est moins un paradis qu'une suspension de l'enfer; c'est un arrêt des combats, une trêve au cours de laquelle on s'adonne aux plaisirs de l'amitié; c'est une idylle provisoire, soustraite au tourbillon des affaires et des passions; c'est un petit bonheur conquis sur le malheur public.

23 L'écriture du conte comme celle du roman repose sur une exacte séparation des sphères privée et publique. Ici le bien, là le mal. Or, comme l'ont montré les historiens $\mathrm{du} \mathrm{xx}^{\mathrm{e}}$ siècle, cette frontière traditionnelle est mise à mal par les revendications des philosophes et par l'émergence d'une sphère impure, à la fois privée et publique, que Reinhardt Koselleck a décrite comme une expansion publique de l'espace privé: «Espace privé et espace public ne s'excluent donc nullement. Au contraire, la certitude que le for intérieur de la morale a de lui-même réside en son aptitude à devenir public. L'espace privé se dilate lui-même jusqu'à devenir public et c'est seulement dans cet espace public que les opinions privées s'érigent en $\operatorname{loi}^{9}{ }^{9}$.

Comment Marmontel vit-il cette politisation de la sphère privée? Comme une intrusion et une corruption. Comme l'intrusion d'Ariste dans la joyeuse société du Philosophe soidisant, Ariste qui sera finalement chassé quand il aura montré à tous sa véritable noirceur. C'est alors que le rire est légitime, quand il restaure les frontières, quand il dissipe la dangereuse confusion des sphères.

L'échec d'Ariste-Rousseau est celui de la parole trompeuse, rejetée hors de l'espace amical où règnent la légèreté $d u$ jeu et les charmes de la sincérité. C'est, pourrions-nous dire, un rappel de ce qu'est la littérature selon Marc Fumaroli ${ }^{10}$, c'est-à-dire une suspension de la violence rhétorique exercée par l'orateur sur son auditoire, ou encore la conversion du rhéteur au plaisir de la contemplation, grâce auxquelles l'homme découvre que le bonheur réside dans l'accord avec lui-même et dans une vertueuse concorde avec autrui. C'est ce charme que compromettent les responsabilités nouvelles qui incombent aux philosophes à partir de 1750; c'est ce charme auquel Marmontel accorde un répit en tenant à distance du conte l'ironie militante d'un Voltaire. Dans la fiction, ce danger est représenté par tout personnage faisant un usage belliqueux du langage: la parole séductrice du libertin, l'injonction du despote oriental et la supercherie ourdie par un mari artificieux sont autant de manifestations d'une violence qui gronde et qui menace à chaque ligne le doux foyer du conte moral.

Nous comprenons pourquoi l'on rit peu dans les contes moraux de Marmontel, pourquoi l'on rit sans éclat, modérément, car il ne faut pas réveiller le diable qui sommeille et attend son heure pour semer le trouble, comme le serpent mordant Myrtis dans Palémon: «Alors, se dépouillant de sa tunique nuptiale, il s'était plongé dans les eaux du lac [...] mais lorsqu'il en sortit, aussi pur, aussi éclatant que la feuille du lys ou celle du narcisse, lorsqu'elle brille encore de la rosée du matin, un énorme serpent, qui se tenait cachée sous l'herbe, et qui se sent foulé sous les pieds de Myrtis, se dresse, l'enveloppe, se replie autour de son corps ${ }^{11} »$. On rit peu, parce que l'on tremble; on rit peu, parce que l'on se sait faible, parce que le spectacle de la souffrance d'autrui renvoie le lecteur sa propre fragilité.

Tout à coup dans les airs un effroyable cri s'élève. Ma troupe et moi nous l'entendons de loin, et saisis de frayeur, nous écoutons. Le cri redouble, et nous voyons un groupe de pasteurs, plus voisin du lac, lever les mains au ciel, et par ses mouvements, exprimer l'horreur et l'effroi. C'était Myrsis que l'on voyait enceint des longs replis de ce serpent qui l'étouffait. Hélas! ma fille et ses compagnes 
n'avaient pas même entendu ses cris; et tandis que le malheureux s'épuisait en efforts pour se dégager de ces nœuds, dont il était comme enchaîné, ma fille, son amante, ivre de bonheur et de joie, et le front couronné de fleurs, dansait au fond de la prairie, et animait par son exemple un cercle de jeunes amants. ô trompeuse prospérité! qui peut se fier à tes caresses? qui peut s'endormir dans ton sein? ${ }^{12}$

On rit peu, parce que l'on a perdu l'illusoire assurance de la force, une force que l'on a troquée contre le sentiment d'une fraternité souffrante et vertueuse. On rit peu, parce que l'heure est à l'engagement public qui enjoint à l'écrivain de quitter la douceur du conte moral pour la violence d'un combat contre l'intolérance religieuse et l'oppression politique.

Encore une fois, faisons bien attention de ne pas forcer le trait, car le conte moral est moins un refus de la politisation de la littérature que sa lente et progressive conversion à la propagande philosophique. Le rire a une fonction régulatrice au cours de cette mutation, comme dans Le Philosophe soi-disant où il sanctionne un mésusage de la parole philosophique; il a sa place dans le conte moral, mais on peut dire que, de façon générale, il n'est pas le bienvenu: dangereux dans la sphère privée fondée sur la solidarité compassionnelle, il est inconvenant dans la sphère publique où le public préside un tribunal de la vertu et du mérite. On ne rit plus d'Alceste corrigé, car la communauté ne se pense plus au XVIII ${ }^{\mathrm{e}}$ siècle, comme elle le faisait au XVII ${ }^{\mathrm{e}}$, sur le mode de la fragmentation et de la hiérarchisation sociale si propices à la rupture comique, mais sur celui de l'égalité des individus et de la communauté des consciences. C'est l'avis de Louis de Sacy, auteur en 1715 d'un Traité de la gloire:

Le concours de tous ces témoignages particuliers que chacun rend en secret aux vertus distinguées, et aux talents reconnus, forme le suffrage public, qui n'est ni moins libre, ni moins sincère, et de ce suffrage naît cette gloire pure, et légitime, dont malgré l'envie et la malignité, brillent la plupart des grands hommes pendant leur vie, et qui consacre en quelque sorte leur mémoire après leur mort ${ }^{13}$.

L'opinion publique n'est pas la manifestation bruyante d'un parterre de rieurs, mais l'émanation rationnelle et sensible d'une société fraternelle de juges d'autant plus intègres qu'ils sont susceptibles un jour de devoir à leur tour se présenter devant le même tribunal. Cette réversibilité, qui est un trait majeur de l'opinion publique qui voit le jour au XVIII ${ }^{\mathrm{e}}$ siècle, est incompatible avec le dispositif comique qui accorde au rieur l'assurance d'une parfaite inamovibilité de sa position.

Le conte moral n'est pas un «conte à rire», parce que le rire y est irrecevable, d'abord moralement, quand il jette un soupçon d'immoralité sur un récit qui fonde sa moralité sur la compassion vertueuse, ensuite politiquement, quand Marmontel appréhende la communion publique des consciences sur un mode pathétique, c'est-à-dire quand il désigne la vertu publique comme une vertu privée politisée, et l'opinion comme l'expansion publique de la conscience morale.

Cette double dissidence, morale et politique, du rire dans les contes et romans de Marmontel pose un problème concernant la catégorisation générique de ces récits et tout particulièrement celle du conte moral, à propos duquel on est en droit de se demander s'il est vraiment un conte. 


\section{Le conte moral est-il un conte?}

provoqué la perplexité. Certains critiques modernes les trouvent bien peu moraux, tandis que d'autres affirment que ce sont de mauvais contes. Christiane Mervaud et Frédéric Deloffre estiment que «ces contes prétendus moraux ne le sont guère» tandis que Henri Coulet déclare que «les contes sentimentaux et moralisants unissent le ridicule à la fausseté», mais la plus violente critique est formulée par Jean Fabre qui affirme que «le pire danger qui menace un conte, le défaut qui condamnait d'avance la prétention du siècle dans l'art du conte» est «celui de devenir un conte moral». D'autres critiques enfin ont émis des doutes sur le fait même qu'il s'agisse de véritables contes, comme Jean Sgard qui y voit une «forme mouvante ${ }^{14}$ », et Henri Coulet ${ }^{15}$ une «expression figée» sans pertinence, sous laquelle les écrivains ont rangé toutes sortes de productions. Le conte moral résulterait, comme beaucoup d'autres formes littéraires de l'époque, d'une pratique de l'hybridation. Si tel était le cas, il nous faudrait conclure, avec Jean Sgard, que le conte moral est une hybridation ratée, une expérience malheureuse au cours de laquelle Marmontel aurait méconnu, négligé ou écarté, ce qui fait la valeur de chaque substance entrant dans la composition du conte moral, à commencer par ce qui fait la valeur du conte: sa gaieté. Il n'est cependant plus possible de considérer, comme on l'a fait, les contes moraux comme de mauvais contes, mais bien plutôt comme l'expression moderne d'une forme plus ancienne que nous aurons à déterminer et que nous ne déterminerons qu'en tenant compte de son incompatibilité avec le rire.

Plusieurs hypothèses ont été avancées à propos d'une filiation entre le conte moral et des genres antérieurs. Jean-Paul Sermain, par exemple, désigne le conte moral comme un héritier du conte de fées, mais c'est aussi un conte qui prétend en finir avec la féerie traditionnelle en imposant, contre le merveilleux surnaturel des fées, un merveilleux naturel qui prend chez Marmontel la formes de prodiges moraux: «Si la nature, s'éloignant de ses sentiers battus, produit un composé moral ou physique assez étrange, pour nous persuader qu'elle y a mis une expresse intention de se surpasser elle-même, ou de ne pas se ressembler, ce procédé, dont les moyens nous sont inconnus, nous étonne, et devient un prodige à nos yeux ${ }^{16} »$. Le conte moral ne serait donc que l'exposition narrative de prodiges vertueux. Pas ou peu de gaieté, mais, comme le précise Marmontel dans son article «Conte» des Élements de littérature, une visée morale qui assure l'unité du récit:

L'unité n'est pas aussi sévèrement prescrite au conte qu'à la comédie; mais un récit qui ne serait qu'un enchaînement d'aventures, sans une tendance commune qui les réunît en un point, serait un roman, et non pas un conte. [...] Quant à la moralité, quoiqu'on en fasse pas au conte une loi rigoureuse, il doit pourtant, comme la comédie, avoir son but, s'y diriger comme elle, et comme elle y atteindre: rien ne le dispense d'être amusant, rien ne l'empêche d'être utile; il n'est parfait qu'autant qu'il est à la fois plaisant et moral ${ }^{17}$.

Ce qui n'est ici qu'une recommandation théorique devient un principe intangible du conte moral qui prend deux formes. Soit Marmontel formule d'entrée une sentence morale, concise ou développée, dont la narration subséquente sera l'exacte illustration fictionnelle, comme c'est le cas dans La Mauvaise Mère qui commence ainsi:

Parmi les productions monstrueuses de la nature, on peut compter le cœur d'une mère qui aime l'un de ses enfants, à l'exclusion de tous les autres. Je ne parle point

Féeries, 5 | 2008 
d'une tendresse éclairée qui distingue entre ces jeunes plantes qu'elle cultive, celle qui répond le mieux à ses premiers soins; je parle d'une tendresse aveugle, souvent exclusive, quelquefois jalouse, qui se choisit une idole et des victimes parmi ces innocents qu'on amis au monde, et pour qui l'on est également obligé d'adoucir le fardeau de la vie. C'est de cet égarement si commun et si honteux pour l'humanité, que je vais donner un exemple ${ }^{18}$.

Soit, à l'inverse, la narration vient mourir sur l'énonciation d'une clausule morale, comme c'est le cas dans Heureusement où un abbé se reconnait dans le portrait que fait la marquise d'un homme pour lequel elle fut sur le point jadis d'accorder ses faveurs:

- Hélas, ce petit imbécile, c'était moi! - Vous il n'est pas possible! - C'était moimême, rien n'est plus certain. Vous me rappelez mon histoire. Ah! cruelle, si j'avais su ce que je sais! - Mon vieil ami, vous auriez eu trop d'avantage, et cette sagesse que vous vantez tant vous eût faiblement résisté. Je suis confondu, s'écriait l'abbé: je ne me le pardonnerai de ma vie. Consolez-vous, il en est temps, reprit en souriant la marquise; mais avouez qu'il y a bien souvent du bonheur dans la vertu même, et que celle qui en ont le plus devraient juger moins sévèrement celles qui n'en ont pas assez ${ }^{19}$.

Dans l'un et l'autre cas, la poétique du conte moral interdit toute liberté narrative au récit, en ce sens que toute digression devient un écart immoral. Il en va de même du rire: soit il se dégrade en rictus moral, soit il est interdit parce que perçu comme une contestation de l'autorité morale du précepte à illustrer.

Nous comprenons mal aujourd'hui cette restriction morale de la narration et du comique, mais si le conte moral plut aux lecteurs du XviII siècle, et on sait qu'il plut, ce n'est pas en raison de ses qualités comiques et narratives, mais en vertu d'autres charmes qu'il faut chercher ailleurs, dans d'autres formes d'écriture et, en l'occurrence, dans la description.

Nous disons en effet que le conte moral est moins un récit qu'une peinture morale, et que Marmontel fut plus sûrement le continuateur de La Bruyère que celui de Molière. Pour s'en convaincre, revenons un instant sur l'article «Conte» de ses Éléments de littérature et à la définition marmontélienne du conteur: «Le conteur doit décrire et peindre, rendre présent aux yeux de l'esprit le lieu de la scène, la pantomime et le tableau de l'action; mais dans le choix de ces détails il ne doit s'attacher qu'à ce qui intéresse ou la vraisemblance ou les mœurs ${ }^{20}$ ».

Le conteur est un peintre des mœurs; est-ce à dire que les contes moraux sont des caractères? est-ce à dire que le comique moral des contes est l'héritier du comique des Caractères? On sait bien qu'il n'en est rien, avant même de se donner la peine d'y réfléchir. Il est certes possible de réunir caractères et contes moraux sous la même bannière de l'éthopée narrativisée, c'est-à-dire la mise en récit de caractères moraux, mais La Bruyère et Marmontel font un usage parfaitement contraire de cette forme: alors que la Bruyère brosse le portrait d'êtres dans lesquels le lecteur est sommé de se reconnaître, Marmontel ne lui présente que des êtres exemplaires qu'il est réduit à admirer. Il s'agit pour Marmontel de construire des icônes sans vraisemblance tant les images sont vertueuses, tant Marmontel est soucieux de creuser l'écart qui sépare le commun de ses prodiges de vertu. Son ambition est de porter l'admiration à son comble, de reproduire sur un mode laïc la relation qui existe entre le fidèle et le saint chrétien. Le conte moral offre aux simples mortels des exemples naturels de plénitude corporelle et spirituelle, des exemples d'une humanité portée à son plus haut degré de perfection morale. La Bruyère raillait et inquiétait, avec son humanité désarticulée, 
éclatée "en une multitude de choses concrètes que ne relie aucun souffle d'âme»; Marmontel, au contraire, édifie et rassure, brossant en plein le portrait radieux d'un homme moral que La Bruyère ne décrivait qu'en creux.

On comprend pourquoi Marmontel s'accommode mal du rire: le comique a depuis toujours fait mauvais ménage avec l'exemplarité religieuse et morale. L'article «Ris ou Rire» de l'Encyclopédie nous le rappelle: «Saint Basile condamne le rire dans tous les Chrétiens sans exception, sur ce passage de l'Écriture, malheur à vous qui riez, parce que vous pleurerez, Luc, VI, ch. xxv». Or, comme l'avait suggéré Angus Martin ${ }^{21}$, le conte moral relève de la liturgie des saints, ou plus exactement d'une esthétique légendaire adaptée aux Lumières. Marmontel nous le souffle dans L'École de l'amitié où il est question d'Alcime:

Il avait quelque ressemblance avec sir Grandisson: comme lui vertueux, modérément sensible, sage dans tous ses goûts, incorruptible dans ses mœurs, possédant son âme et ses sens dans une paix inaltérable, Alcime était, dans sa jeunesse, l'homme du monde le plus considéré. Dans le petit nombre des sociétés dont il avait fait son choix, on le citait comme un modèle, on l'écoutait comme un oracle $^{22}$.

41 Le propos est corroboré par un passage de l'Essai sur les romans où il est question de sir Grandisson:

Dans ce caractère, rare et merveilleux par l'accord de ses qualités réunies, tout est simple, aisé, naturel, sans ostentation, sans effort; dans cette élévation d'âme il n'y a rien d'outré; [...] dans cette conduite, toujours si noble et si généreuse, il n'y a pas un trait de romanesque; [...] dans les situations critiques et les conjonctures délicates où se trouve ce personnage, ce n'est jamais qu'un homme de bien, tel qu'il est possible à chacun de l'être, si, avec une saine raison, l'on se sent doué d'un bon cœur. Ce n'est donc qu'avec de la bonté, de la droiture, du courage, et un juste mélange de sensibilité, de force, et de douceur, que ce modèle est composé: il en résulte cependant un ensemble si admirable, qu'avec les simples qualités d'un homme, sir Charles Grandisson est comme un dieu à qui l'on rend une espèce de culte, et pour qui l'amour le plus pur, le respect le plus tendre, la vénération la plus profonde et la plus unanime, n'ont rien que de très juste et de très naturel ${ }^{23}$.

Le rire est indésirable dans les contes comme il l'est dans le sermon, parce qu'il relève sourdement d'un blasphème perpétré à l'endroit des nouvelles idoles de la philosophie des Lumières. À bien y réfléchir, Voltaire et Marmontel, le maître et l'émule, sont complémentaires: l'un brise les idoles anciennes, pendant que l'autre en élève de nouvelles, conformes aux exigences naturalistes du siècle. Qui croirait aujourd'hui que les contes moraux eurent au $\mathrm{XVIII}^{\mathrm{e}}$ siècle plus de succès que les contes de Voltaire? La postérité a donné raison au rire sur les larmes, au rire qui n'a de place que dans la dénonciation iconoclaste des saintes effigies. Voltaire démystifie avant que Marmontel n'édifie.

\section{Le rire immoral}

Tout le monde n'applaudit cependant pas au beau tandem philosophique. Si le maître et son disciple ont l'ambition de substituer une nouvelle exemplarité philosophique à l'exemplarité religieuse défaillante, ils vont bientôt devenir à leur tour l'objet de quolibets. Marmontel surtout apparaît comme une risible autorité que malmènent les conteurs immoralistes. On rit peu dans les contes moraux, mais on rit beaucoup des contes moraux dans les «contes anti-moraux» et dans les «contes immoraux» du prince 
de Ligne, mais aussi dans les nombreux contes que leurs auteurs, à l'instar de Crébillon fils avec son Sopha, ont affublé de la très marmontélienne étiquette "conte moral»: Voltaire avec sa Bégueule, Dorat avec son Combabus, Mérard de Saint-Just avec sa Doris...

Le rire chassé du conte moral par le pathétique va se réfugier dans le conte immoral. Notons bien toutefois que le conte moral n'est pas qu'un conte anti-moral, parce qu'il déborde le seul cadre contestataire de l'anti-moralité. Il prend au XVIII siècle trois formes majeures. C'est d'abord, au tournant des XVII et XVIII ${ }^{\mathrm{e}}$ siècles, un conte en vers écrit sous le patronage de La Fontaine et par des conteurs comme Grécourt, Piron et Vergier. C'est ensuite, pendant la première moitié $d u$ xvIII ${ }^{e}$ siècle, un conte de fées où les écrivains se plaisent à mettre en scène des fées lascives et des génies lubriques: Crébillon fils et Voisenon en offrent les plus savoureux exemples. C'est enfin, au tournant des XVIII ${ }^{\mathrm{e}}$ et XIX ${ }^{\mathrm{e}}$ siècles, un conte réaliste où des conteurs comme Nerciat, Sade et Ligne, choisissent la société d'Ancien Régime pour scène de l'immoralité moderne.

On rit dans les contes immoraux, mais pour des raisons différentes, et surtout on constate que, à l'instar de ce qui se passe dans les contes moraux, on y rit de moins en moins, des contes en vers aux contes de fées, et moins encore des contes de fées aux contes réalistes. On rit toujours moins, parce que la nature de l'immoralité des contes a changé. Le rire immoral connaît en effet trois âges, qui correspondent aux trois variétés de contes immoraux $\mathrm{du} \mathrm{xvIII}^{\mathrm{e}}$ siècle. C'est d'abord une immoralité oppositionnelle, où le désordre immoral se produit en marge d'un ordre moral puissant: on rit du larcin d'une jeunette dépucelée, d'une épouse infidèle ou d'un curé malicieux. C'est ensuite une immoralité substitutive, où la féerie va permettre au conteur d'inventer un nouvel ordre immoral qui est bien souvent la parfaite inversion de l'ordre moral réel: on rit d'un monde où le vice est ouvertement exalté et la vertu unanimement condamnée. On rit, mais on s'inquiète aussi de voir que tout ordre est relatif, et que le vice peut, comme la vertu, servir de principe fondateur à un ordre social alternatif. C'est enfin une immoralité problématique, où le conteur s'applique à effacer les frontières entre ordre moral et désordre immoral, de façon à révéler la part d'immoralité présente dans tout ordre moral, ainsi que la part de moralité dans tout comportement immoral. On ne rit plus guère quand on a perdu ses certitudes morales. On ne rit plus, mais peut-être est-on alors en face d'un véritable conte moral, c'est-àdire en face d'un conte qui, comme ceux de Diderot, met en évidence les apories du jugement moral.

Qu'ils soient moraux ou immoraux cependant, les contes cessent toujours plus d'être des «contes à rire», soit, comme les contes moraux, parce qu'ils œuvrent à fonder une nouvelle exemplarité sur des fondements naturels, soit, comme les contes immoraux, parce qu'ils mettent en péril la validité du jugement moral. Chez les uns comme chez les autres conteurs, le rire a laissé place au rictus. 


\section{NOTES}

1. Le conte, s'il n'a pas sa place dans les arts poétiques, fait en revanche l'objet de recherches historiques. Marmontel dans ses Éléments de littérature, Dorat dans ses Réflexions sur le conte et Gudin de La Brenellerie dans son Histoire sur l'origine des contes font défiler les différents maîtres conteurs. Pour lors, ils affirment, comme Dorat en 1765, la supériorité de l'approche historique sur la prescription poétique : "L'étude des grands modèles vaut mieux que toutes les leçons de nos prétendus législateurs. Horace, Vida et Boileau réunis ne formeront jamais un poète : ils ont mis en vers de belles et d'harmonieuses inutilités. C'est par ses fautes et ses écarts que le génie s'éclaire. Ainsi, sans vouloir donner des leçons, je hasarderai mon sentiment sur le genre de conte que je crois le plus fait pour réussir parmi nous." Sur cette question, voir Le Conte merveilleux au XVIII ${ }^{e}$ siècle. Une poétique expérimentale, textes réunis par R. Jomand-Baudry et J.-

F. Perrin, Paris, Kimé, 2002.

2. Dictionnaire de l'Académie, art. «Conte », Paris, 1694, p. 239.

3. Marmontel, Éléments de littérature, art. « Conte », Paris, Desjonquères, 2005, p. 304.

4. Ibid., p. 303.

5. Marmontel, Contes moraux, Paris, Merlin, 1765, « Préface », p. I-II.

6. Nous citons le texte de l'article réécrit et publié dans ses Éléments de littérature, ouvr. cité, p. 290.

7. Anne Coudreuse, Le Goût des larmes au XVIII siècle, Paris, PUF, 1999, p. 79-80.

8. Voir Keith Michael Baker, "L'opinion publique comme invention politique ", dans Au tribunal de l'opinion. Essais sur l'imaginaire politique au XVIII siècle, Paris, Payot, 1993, p. 219-265. Voir également notre discussion de la thèse de Baker dans Nicolas Veysman, Mise en scène de l'opinion publique dans la littérature des Lumières, Paris, Honoré Champion, 2004, p. 21-25.

9. Reinhart Koselleck, Le Règne de la critique, Paris, Éditions de Minuit, 1979, p. 46.

10. Voir Marc Fumaroli, "Les sanglots d'Ulysse ", Mesure, 3, 1990, p. 169-183 ; repris dans La Diplomatie de l'esprit. De Montaigne à La Fontaine, Paris, Gallimard, coll. « tel », 1998, p. 1-22.

11. Marmontel, Palémon. Conte pastoral, dans Nouveaux Contes moraux, Leipsic, Lebrecht Crusius, 1805 , t. IV, p. 94.

12. Ibid., p. 94-95.

13. Louis de Sacy, Traité de la gloire, Paris, Huet, 1715, p. 9.

14. Jean Sgard, "Marmontel et la forme du conte moral », dans De l'Encyclopédie à la ContreRévolution. Jean-François Marmontel (1723-1799), édité par Jean Ehrard, Clermont-Ferrand, G. de Bussac, 1970, p. 229 : «En un demi siècle, la formule a évolué ; le conte marmontélien fut tout à tour apologue et comédie-proverbe, anecdote et récit; et pourtant, il s'agit toujours de "contes moraux", c'est-à-dire, comme l'écrit Morellet, d'un "classe à part", d'un "genre". Mais comment définir ce genre malléable et propre, semble-t-il, à son seul auteur? » D'abord perplexe, Jean Sgard engage son travail de définition après avoir conclu qu'« il s'agit [...] de contes » et rattache Marmontel à la famille des conteurs plaisants.

15. Henri Coulet, "Peut-on définir le conte moral ?", dans Narrativa Francesca en el siglo XVIII, Actas de la Jornadas Literarias, 27-29 nov. 1986, éd. A. Yllera et M. Boixareu Vilaplana, Madrid, 1988, p. 37-38: "Il semble que "conte" ait été une expression figée, dans laquelle le terme "moral" entraînait le terme "conte": parce qu'ils étaient rangés, initialement ou secondairement, dans le genre moral, on a appelé "contes" un poème épique en prose [...], une "pièce dramatique en cinq actes" [...], un drame en trois actes [...], un roman par lettres [...], de longs romans et toutes les variétés de récit court [...]. »

16. Marmontel, Poétique française, chap. $X$.

17. Marmontel, Éléments de littérature, art. « Conte », ouvr. cité, p. 303. 
18. Marmontel, Contes moraux, Paris, Merlin, 1765, t. II, p. 93.

19. Ibid., t. I, p. 284.

20. Marmontel, Éléments de littérature, art. "Conte », ouvr. cité, p. 302.

21. Voir Angus Martin, «Présentation » dans Anthologie du conte en France. Philosophes et cœurs sensibles, Paris, UGE, « 10/18 », 1981, p. 14.

22. Marmontel, L'École de l'amitié, dans Nouveaux Contes moraux, ouvr. cité, t. II, p. 6.

23. Marmontel, Essai sur les romans considérés du côté moral, dans Euvres complètes, Genève Slatkine reprints, 1968 , tome III, $2^{\mathrm{e}}$ partie, p. 585-586.

\section{RÉSUMÉS}

De l'avis des lexicographes et des théoriciens du genre, le conte serait toujours un « conte à rire ». C'est l'avis de Marmontel, père du conte moral, et en effet ses premiers contes peuvent être tenus comme des « contes à rire » ; mais au fil des récits le comique se révèle toujours plus inconciliable avec la moralité, et les contes moraux, après 1758 , sont bien plutôt des « contes à pleurer ». Cette éviction du rire nous conduit à douter de l'inscription générique des contes moraux, des contes que nous caractériserons comme de faux contes et comme de vraies éthopées. On s'imaginera que le rire est alors rejeté dans l'« antipode de Marmontel ", dans les contes immoraux du xviIIesiècle, et l'on aura raison, mais on remarquera, des contes en vers aux contes de fées libertins et jusqu'aux contes immoraux de la fin du siècle, le même recul du rire, le même rictus.

Marmontel's moral rictus

According to lexicographers and theorists of the genre, all tales are "tales-for-laughter". Such was Marmontel's opinion, and the first works by this founding father of moral tales were indeed tales-for-laughter, but the comical element became less and less compatible with morality, and after 1758, moral tales are rather to be read as "tales-for-tears". This elimination of laughter leads us to question the genre of moral tales, which therefore appear as false tales, but as true ethopeias. Laughter thus appears as rejected to Marmontel's antipodes, into the immoral tales of the 18th century, even though the same retreat of laughter, the same rictus, can be observed in verse tales, in fairy tales and in the immoral tales of the end of the century themselves.

\section{AUTEUR}

\section{NICOLAS VEYSMAN}

Paris 